\title{
Characterisation of indeterminate focal breast lesions on grey-scale ultrasound: role of ultrasound elastography
}

\section{Caratterizzazione delle lesioni focali mammarie indeterminate all'ecografia in scala di grigi: ruolo della elastosonografia}

\author{
T.V. Bartolotta ・ R. Ienzi • A. Cirino • C. Genova ・ F. Ienzi ・ D. Pitarresi ・ E. Safina ・ M. Midiri \\ Dipartimento di Biotecnologie Mediche e Medicina Legale, Sezione di Diagnostica per Immagini, Policlinico Universitario, Via del \\ Vespro 127, 90127 Palermo, Italy \\ Correspondence to: T. Bartolotta, Tel.: +39-091-6552335/6552336, Fax: 39-091-6552337, e-mail: tv.bartolotta@unipa.it
}

Received: 22 June 2010 / Accepted: 30 August 2010 / Published online: 19 March 2011

(C) Springer-Verlag 2011

\begin{abstract}
Purpose. This study was undertaken to evaluate the role of ultrasound (US) elastography in characterising focal breast lesions classified as indeterminate on B-mode US.

Materials and methods. Eighty-four focal breast lesions, 64 benign and 20 malignant (mean diameter, $15.1 \mathrm{~mm}$ ), detected but not characterised on B-mode US in 72 women, Breast Imaging Reporting and Data System (BI-RADS) US category $3(n=56)$ or category $4(n=28)$, were studied with US elastography and classified in consensus by two radiologists according to a five-point colour scale. Sensitivity, specificity and positive and negative predictive values (PPV and NPV) of US elastography compared with conventional US were calculated in relation to microhistology $(n=67)$ and cytology $(n=17)$, which were used as the reference standard.

Results. A total of 65/84 (77.4\%) lesions were correctly classified as benign or malignant using US elastography, whereas the remaining 19/84 (22.6\%) were incorrectly assessed. There were no statistically significant differences between US elastography and B-mode US with regard to sensitivity (70\% vs. 68.4\%), specificity (79.6\% vs. $78.5 \%)$, PPV (51.8\% vs. $48.1 \%$ ) and NPV $89 \%$ vs. $89.5 \%$ ( $p>0.5)$. By contrast, a statistically significant difference was noted in the evaluation of BI-RADS 3 lesions, in which US elastography had 50\% sensitivity, $86 \%$ specificity, $30 \%$ PPV and $93.5 \%$ NPV compared with BI-RADS 4 lesions $(78.6 \%, 57.1 \%, 64.7 \%$ and $72.7 \%)(p<0.5)$.

Conclusions. The high NPV of US elastography may help reduce the use of biopsy in BI-RADS 3 lesions, but its low PPV in BI-RADS 4 lesions does not allow avoidance of
\end{abstract}

\section{Riassunto}

Obiettivo. Scopo del nostro lavoro è stato valutare il ruolo dell'elastosonografia nella caratterizzazione delle lesioni focali mammarie indeterminate all'ecografia di base. Materiali e metodi. Ottantaquattro lesioni focali mammarie, 64 benigne e 20 maligne (diametro medio: $15,1 \mathrm{~mm}$ ), rilevate ma non caratterizzate all'ecografia di base in 72 donne - punteggio ecografico breast imaging reporting and data system (BI-RADS) $3(n=56)$ o BI-RADS $4(n=28)$-, sono state sottoposte ad elastosonografia e classificate in consenso da due radiologi secondo una scala colorimetrica in cinque punti. Sono stati calcolati sensibilità, specificità, valori predittivo positivo $(V P P) e$ negativo (VPN) dell'elastosonografia rispetto all'ecografia convenzionale in relazione alla metodica di riferimento: microistologia $(n=67)$ e citologia $(n=17)$.

Risultati. Sessantacinque/84 (77,4\%) lesioni sono state correttamente classificate come benigne o maligne all'elastosonografia, mentre le rimanenti 19/84 (22,6\%) sono state erroneamente valutate. Non sono state riscontrate differenze statisticamente significative tra elastosonografia ed ecografia B-mode con tassi di sensibilità, specificità, VPP e VPN pari rispettivamente al $70 \%, 79,6 \%, 51,8 \%$ e $89 \%$, e al 68,4\%, 78,5\%, 48,1\% e $89,5 \%(p>0,5)$. L'elastosonografia ha presentato differenze statisticamente significative nella valutazione delle lesioni mammarie in classe BI-RADS 3, con valori pari a 50\%, $86 \%, 30 \%$ e $93,5 \%$, rispetto a quelle BI-RADS $4(78,6 \%$, $57,1 \%, 64,7 \%$ e $72,7 \%)(p<0,5)$.

Conclusioni. L'elevato VPN dell'elastosonografia può essere utile per evitare l'estensivo ricorso alla biopsia nelle lesioni mammarie in classe BI-RADS 3, ma il basso 
biopsy on the basis of the US elastographic score alone in this group of lesions.

Keywords Breast · Ultrasound elastography $\cdot$ Breast neoplasms
$V P P$ in quelle BI-RADS 4 non consente di escludere il ricorso alla biopsia sulla scorta del solo punteggio elastosonografico.

Parole chiave Mammella $\cdot$ Elastosonografia $\cdot$ Neoplasie mammarie

\section{Introduction}

The role of grey-scale ultrasound (US) has considerably expanded in characterising focal breast lesions. This is a result of continuous technological advances that have greatly improved both its spatial and contrast resolution [1]. In particular, the development of a categorisation system based on sonographic semiological criteria, such as the Breast Imaging Reporting and Data System (BI-RADS), has proved useful for describing and classifying both benign and malignant breast lesions [2]. However, the limited reproducibility of the system in determining the exact likelihood of malignancy of suspicious breast lesions (category 4 ), with variability ranging from $3 \%$ to $94 \%$, leaves the problem of recourse to more invasive and costly diagnostic procedures, such as biopsy, essentially unchanged [3]. Furthermore, even though the risk of malignancy in lesions classified as BI-RADS category 3 should be $<2 \%$, the large number of biopsies performed on benign lesions continues to represent a real problem, especially in the light of the cost-effectiveness of screening programmes [2].

The introduction of US elastography into clinical breast imaging in 1997 made it possible to evaluate a mechanical property completely different from echogenicity: that is, tissue elasticity [4]. Tissue elasticity, defined mathematically by the elastic or Young modulus, can be obtained from the pressure required to deform a given tissue or structure [5]. The recent availability of US units equipped with an elastography module enabling real-time, free-hand imaging has prompted several authors to assess the contribution of US elastography to the characterisation of breast lesions, with encouraging results [6-10]. The purpose of this study was to evaluate the usefulness of US elastography in characterising breast lesions classified as indeterminate at B-mode US.

\section{Materials and methods}

Patients

Between June 2008 and June 2009, 72 women (age range 22-84 years; mean age, $47.5 \pm 12.33$ years) with 84 focal breast lesions (diameter, 4-44 mm; mean, $15.1 \mathrm{~mm}$ ) were prospectively evaluated following approval by the ethics

\section{Introduzione}

L'ecografia in scala di grigi, grazie ai continui progressi tecnologici, che ne hanno notevolmente migliorato sia la risoluzione spaziale sia quella di contrasto, ha notevolmente ampliato il suo ruolo nella caratterizzazione delle lesioni focali mammarie [1]. In particolare, l'elaborazione di un sistema di categorizzazione basato su criteri semeiologici ecografici, quale il breast imaging reporting and data system (BI-RADS), si è dimostrato uno strumento utile ai fini di descrivere e sistematizzare le lesioni mammarie sia benigne che maligne [2]. Tuttavia, la limitata riproducibilità di tale sistema nel definire l'esatta probabilità di malignità di una lesione mammaria sospetta (categoria 4), con una variabilità che va dal 3\% al 94\%, lascia sostanzialmente inalterata la problematica relativa al ricorso, in tale ambito, a procedure diagnostiche più invasive e costose, quali la biopsia [3]. Inoltre, sebbene il rischio di malignità delle lesioni classificate in categoria BI-RADS 3 dovrebbe essere inferiore al $2 \%$, il gran numero di biopsie tutt'oggi effettuate su lesioni benigne continua a costituire un reale problema, soprattutto nell'ottica costo/beneficio dei programmi di screening [2].

L'introduzione in ambito clinico-senologico dell'elastosonografia, avvenuta nel 1997, ha reso possibile valutare una proprietà meccanica completamente differente dall'ecogenicità: l'elasticità tissutale [4]. Quest'ultima, definita matematicamente dal modulo elastico o di Young, può essere ricondotta alla pressione necessaria per deformare un dato tessuto o struttura [5]. Del tutto recentemente, la disponibilità commerciale di unità ecografiche dotate di modulo elastosonografico capace di consentire un esame in tempo reale ed a mano libera, ha consentito a diversi autori di cominciare a valutare l'apporto dell'elastosonografia nella caratterizzazione delle lesioni mammarie con incoraggianti risultati [6-10]. Scopo del presente lavoro è stato quello di valutare l'utilità dell'impiego dell'elastosonografia nella caratterizzazione delle lesioni mammarie indeterminate all'ecografia di base.

\section{Materiali e metodi}

Pazienti

Ottenuta l'approvazione da parte del comitato etico istituzionale, sono state prospetticamente valutate, nel periodo 
committee. All lesions were visible on US. Thirty-seven were also clinically palpable (diameter, $8-44 \mathrm{~mm}$; mean, $20.9 \mathrm{~mm}$ ), whereas 47 lesions (diameter 4-15 mm; mean, $8 \mathrm{~mm}$ ) were nonpalpable and were detected during routine examinations. In particular, 49 patients had previously undergone mammography. All patients underwent US investigation conducted by two radiologists, each with a minimum of 10 years' experience in breast US, who classified each lesion according to the BI-RADS US classification [11]. All lesions categorised as BI-RADS 3 and 4 were further investigated by US elastography during the same session. In order to assess the impact of elastography on the diagnostic workup, all lesions with a clear cystic appearance and/or classified as BI-RADS 2 (and therefore not requiring biopsy) were excluded from the analysis. The lesions classified as BI-RADS 5 were also excluded, as they were almost certainly malignant and requiring appropriate treatment.

Examination technique

All investigations were performed using Logos Hi Vision Gold US equipment (Esaote/Hitachi Medical Corporation, Tokyo, Japan) equipped with a multifrequency linear-array transducer (13-5 MHz) and a dedicated US elastography module. Before the US elastography examination itself, the breast parenchyma was always imaged with grey-scale and colour power Doppler US to locate the lesion and select the most appropriate scan plane. Once adjusted, the technical parameters of the equipment - number and location of focuses and gain - were left unchanged for the duration of the examination. In particular, the US elastography investigation was conducted in real time using a split-screen display with simultaneous viewing of the B-mode image on the right and the US elastogram superimposed on the same B-mode image on the left. The equipment uses a special autocorrelation system (the extended combined autocorrelation method) that can be applied in real time to take into account not only changes along the axis of propagation of the US beam but also movements along the lateral axis, thereby reducing artefacts due to lateral displacement of the tissues [8].

The signal from the structures being examined - and relative to a specific sampling box placed on the region of interest and extending also to healthy breast tissue - is processed in real time and the resulting elastogram is immediately superimposed over the B-mode image and displayed on a colour scale from red (soft or highly elastic tissue) to blue (hard or inelastic tissue). At the same time, a special indicator light provides real-time feedback on the quality of the examination by scoring it from 1 to 6 and displaying the score alongside the US elastogram. Scores of 3 or higher indicate a sufficient or good reliability of the elastogram. compreso tra giugno 2008 e giugno 2009, 72 donne di età compresa tra 22 e 84 anni (età media: 47,5 12,33 anni) con 84 lesioni mammarie focali (diametro: 4-44 mm; media: 15,1 mm), tutte ecograficamente rilevabili. Trentasette lesioni erano anche clinicamente palpabili (diametro: 8-44 mm; media: 20,9 mm), mentre 47 lesioni (diametro 4-15 mm; media: $8 \mathrm{~mm}$ ) non erano palpabili e sono state individuate durante controlli di routine. In particolare, 49 pazienti erano state in precedenza sottoposte ad esame mammografico. Tutte le pazienti, quindi, sono state sottoposte a indagine ecografica, condotta da due radiologi esperti in ecografia senologica (almeno dieci anni), $i$ quali hanno classificato ciascuna lesione, sulla base dei reperti $B$-mode, in accordo con lo schema BI-RADS ecografico [11]. Tutte le lesioni con punteggio BI-RADS compreso tra 3 e 4 sono state avviate, nella stessa seduta, allo studio mirato con modulo elastosonografico. Al fine di valutare l'impatto dell'elastosonografia nel modificare l'iter diagnostico, sono state escluse dalla presente valutazione tutte le lesioni classificate con aspetto chiaramente cistico elo classificate come BI-RADS 2 e, pertanto, non necessitanti di campionamento bioptico. Anche le lesioni classificate come BI-RADS 5 all'indagine ecografica di base sono state escluse dal presente studio, in quanto quasi certamente maligne e già di per sé necessitanti di adeguato trattamento.

\section{Tecnica d'esame}

Tutte le indagini ecografiche sono state effettuate con apparecchiatura Logos HiVision Gold (Esaote/Hitachi Medical Corporation, Tokio, Giappone) dotata di trasduttore lineare multifrequenza (13-5 MHz) nonchè di modulo elastosonografico dedicato. Prima dell'esame elastosonografico vero e proprio, è stato sempre effettuato uno studio ecografico del parenchima mammario, sia in scala di grigi che con modulo color e power Doppler, al fine di individuare la lesione stessa e selezionare un adeguato piano di scansione. Dopo aver effettuato la regolazione dei parametri tecnici dell'apparecchiatura - ad esempio numero e posizione dei fuochi, guadagni - questa è stata mantenuta invariata per tutta la durata dell'esame. In particolare, l'indagine elastosonografica è stata condotta in tempo reale, mediante suddivisione dello schermo ecografico in due parti e visualizzando contemporaneamente nella parte destra dello schermo la consueta immagine B-mode e, nella parte sinistra, l'elastogramma superimposto alla stessa immagine B-mode. L'apparecchiatura impiegata implementa un particolare sistema di autocorrelazione (metodo di autocorrelazione estesa combinata) che può essere applicato in tempo reale permettendo di tenere conto non solo delle alterazioni lungo l'asse di propagazione del fascio ultrasonoro, ma anche dei movimenti lungo l'asse laterale, riducendo gli artefatti dovuti, appunto, alla dislocazione laterale dei tessuti [8]. 
In our study, US elastograms were considered acceptable only if they were found to be adequately and continuously superimposed over the B-mode image, with light indicator scores between 4 and 6 . US elastograms with poor superimposition and with scores $<4$ were considered unreliable. The mean duration of each US elastography study was about 3 (range 2-5) min.

Image analysis

All examinations were digitally stored as raw data. Two radiologists with at least 10 years' experience in breast US and who were not involved in performing the examinations and were unaware of patients' clinical history and final diagnosis, consensually evaluated the images offline. To avoid any recall bias, four image evaluation sessions were scheduled, each 1 week apart.

The US elastograms were classified using a score ranging from 1 to 5 , according to the scheme proposed by the Italian Study Group (elasticity score) based on the classification previously proposed by Itoh et al. [7]: score 1, presence of a three-layered colour pattern of blue, green and red; score 2 , diffuse green, possibly with a few small inconsistent blue spots; score 3, diffuse green with some blue spots; score 4, almost completely blue with maybe a few small green spots, especially peripherally; score 5 , completely blue and with a blue rim surrounding the lesion. A score of 1 is considered predominant in liquid lesions; scores 2 and 3 correspond to lesions that are predominantly elastic and therefore more frequently benign, whereas scores 4 and 5 indicate lesions that are predominantly hard and more frequently malignant $[7,12]$.

\section{Standards of reference}

The final diagnosis was obtained through a microhistological biopsy in 67 cases and cytological examination in the remaining 17. All samples were collected under US guidance using 21- to 23-gauge needles for cytological sampling and 14- to 18-gauge needles for microhistological sampling.

\section{Statistical analysis}

In order to evaluate the usefulness of US elastography in characterising suspicious breast lesions and therefore to verify its sensitivity, specificity and positive and negative predictive values (PPV and NPV), we calculated the true positive (TP), true negative (TN), false positive (FP) and false negative (FN) results. In agreement with the literature, lesions were considered positive $(\mathrm{P})$ for malignancy if they had US elastography scores of 4 or 5 and negative (N) if they had scores of 1,2 or 3 .
Il segnale proveniente dalle strutture esaminate - e relativo ad un apposito box di campionamento posto dall'operatore sulla regione di interesse comprendente anche il tessuto mammario sano - viene elaborato in tempo reale $e$, immediatamente, l'elastogramma ottenuto viene sovrapposto all'immagine in $B$-mode e visualizzato secondo una scala cromatica che va dal rosso (soffice) al blu (anelastico). Al contempo, un apposito indicatore luminoso indica, in tempo reale, la corretta conduzione dell'esame attraverso una scala di valori da 1 a 6 , che viene visualizzata lateralmente all'immagine elastosonografica. Valori uguali o superiori a 3 garantiscono una sufficiente o buona attendibilità dell'immagine elastosonografica. Quest'ultima, nel nostro studio, è stata considerata accettabile solo nel caso in cui fosse risultata sovrapposta in maniera congrua e continua all'immagine B-mode, con valori assegnato dall'apposito indicatore luminoso compresi tra 4 e 6; sono state considerate, invece, inesatte le informazioni provenienti da elastogrammi con una sovraimposizione fugace e con valori di scala inferiori a 4. La durata di ogni singola valutazione elastosonografica è stata, in media, pari a circa 3 minuti (intervallo: 2-5 minuti).

Analisi delle immagini

Tutti gli esami sono stati archiviati come dati grezzi in forma digitale. Due radiologi esperti in ecografia senologica (almeno dieci anni), non coinvolti nell'esecuzione degli esami e non a conoscenza della storia clinica dei pazienti né della diagnosi finale, hanno valutato in consenso off-line le immagini ottenute. Per evitare il recall bias sono state previste quattro sessioni di valutazione distanziate da una settimana.

Le immagini elastografiche sono state classificate con un punteggio variabile da 1 a 5, secondo lo schema proposto dal Gruppo di Studio Italiano (elasticity score) sulla base della classificazione precedentemente proposta da Itoh et al. [7]: (1) presenza di tri-stratificazione cromatica: blu/ verde/rossa; (2) prevalenza di verde, con eventualmente qualche incostante punto blu; (3) prevalenza di verde, con qualche punto blu; (4) quasi completamente blu, con eventualmente qualche punto verde, soprattutto perifericamente; (5) completamente blu, anche con alone blu perilesionale. Il punteggio 1 è considerato predominante nelle lesioni liquide, i punteggi 2 e 3 corrispondono a lesioni in prevalenza elastiche e, dunque, più frequentemente benigne, mentre i punteggi 4 e 5 corrispondono a lesioni prevalentemente rigide e più frequentemente maligne [7, 12].

Standard di riferimento

La diagnosi definitiva è stata ottenuta mediante prelievo microistologico in 67 casi ed esame citologico nei rimanenti 17. Tutti i prelievi sono stati eseguiti sotto guida ecografia 


\section{Results}

The cytohistological examinations of the 84 lesions studied provided a diagnosis of benignity in 64 cases $(76.2 \%)$ and malignancy in the remaining 20 cases $(23.8 \%)$ (Table 1).

Table 1 Final diagnosis and ultrasound (US) elastography score of the 84 breast lesions examined

\begin{tabular}{lcccccc}
\hline US elastography score & 1 & 2 & 3 & 4 & 5 & Total \\
\hline Benign lesions & & & & & & \\
$\quad$ Fibroadenoma & 2 & 21 & 2 & 4 & - & 29 \\
Corpuscular cyst & 6 & 2 & 1 & 2 & - & 11 \\
Angioma & - & 8 & - & - & - & 8 \\
Cicatricial fibrosis & - & 1 & - & 3 & - & 4 \\
Adenosis & - & 1 & 1 & 2 & - & 4 \\
Granuloma & 1 & 2 & - & - & - & 3 \\
Fat necrosis & - & 1 & - & 2 & - & 3 \\
Pseudoangiomatous & - & - & 1 & - & - & 1 \\
hyperplasia & & & & & & \\
Cold abscess & 1 & - & - & - & - & 1 \\
Malignant lesions & & & & & & \\
Invasive ductal & & & 1 & 8 & 3 & 12 \\
carcinoma & & & & & & \\
Ductal carcinoma in situ & - & - & - & 1 & - & 1 \\
Abscessed ductal & 1 & - & - & - & - & 1 \\
carcinoma & & & & & & \\
Tubular carcinoma & - & - & 2 & - & - & 2 \\
Lobular carcinoma & - & - & - & 2 & & 2 \\
Mucinous carcinoma & 1 & - & - & - & - & 1 \\
$\quad$ Sarcoma & - & 1 & - & - & - & 1 \\
Total & 12 & 37 & 8 & 24 & 3 & 84 \\
\hline & & & & & \\
\hline & & & & & &
\end{tabular}

Tabella 1 Diagnosi finale e punteggio elastosonografico delle 84 lesioni mammarie prese in esame

\begin{tabular}{|c|c|c|c|c|c|c|}
\hline $\begin{array}{l}\text { Punteggio } \\
\text { elastosonografico }\end{array}$ & 1 & 2 & 3 & 4 & 5 & Totale \\
\hline \multicolumn{7}{|l|}{ Lesioni benigne } \\
\hline Fibroadenoma & 2 & 21 & 2 & 4 & - & 29 \\
\hline Cisti corpuscolata & 6 & 2 & 1 & 2 & - & 11 \\
\hline Angioma & - & 8 & - & - & - & 8 \\
\hline Fibrosi cicatriziale & - & 1 & - & 3 & - & 4 \\
\hline Adenosi & - & 1 & 1 & 2 & - & 4 \\
\hline Granuloma & 1 & 2 & - & - & - & 3 \\
\hline Steatonecrosi & - & 1 & - & 2 & - & 3 \\
\hline $\begin{array}{l}\text { Iperplasia } \\
\text { pseudoangiomatosa }\end{array}$ & - & - & 1 & - & - & 1 \\
\hline Ascesso freddo & 1 & - & - & - & - & 1 \\
\hline \multicolumn{7}{|l|}{ Lesioni maligne } \\
\hline $\begin{array}{l}\text { Carcinoma duttale } \\
\text { infiltrante }\end{array}$ & & & 1 & 8 & 3 & 12 \\
\hline Carcinoma duttale in situ & - & - & - & 1 & - & 1 \\
\hline $\begin{array}{l}\text { Carcinoma duttale } \\
\text { ascessualizzato }\end{array}$ & 1 & - & - & - & - & 1 \\
\hline Carcinoma tubulare & - & - & 2 & - & - & 2 \\
\hline Carcinoma lobulare & - & - & - & 2 & & 2 \\
\hline Carcinoma mucinoso & 1 & - & - & - & - & 1 \\
\hline Sarcoma & - & 1 & - & - & - & 1 \\
\hline Totale & 12 & 37 & 8 & 24 & 3 & 84 \\
\hline
\end{tabular}

mediante aghi da 21-23 G per l'esame citologico e con aghi da 14-18 G per l'esame microistologico.

\section{Analisi statistica}

Allo scopo di valutare l'utilità dell'impiego dell'elastosonografi a nella caratterizzazione delle lesioni mammarie sospette e quindi di verificarne la sensibilità, la specificità ed il valore predittivo positivo (VPP) e negativo (VPN) abbiamo calcolato: $i$ veri positivi $(V P)$, i veri negativi $(V N)$, i falsi positivi (FP) ed i falsi negativi (FN). Abbiamo considerato, in accordo con i dati della letteratura, positive $(P)$ per malignità le lesioni con punteggio elastosonografico pari a 4 o 5; e negative $(N)$ le lesioni con un punteggio elastosonografico pari a 1, 2 o 3.

\section{Risultati}

L'esame cito-istologico delle 84 lesioni oggetto di studio del nostro lavoro ha fornito una diagnosi di benignità in 64 casi $(76,2 \%)$ e di malignità nei rimanenti 20 casi $(23,8 \%)$ (Tabella 1). All'indagine ecografica di base, 56/84 lesioni $(66,7 \%)$ sono state classificate come appartenenti alla classe BI-RADS 3 e le rimanenti $28(33,3 \%)$ a quella BI-RADS 4. Nelle Tabelle 1-3 sono riportati i punteggi relativi alla valutazione elastosonografica delle lesioni mammarie studiate in rapporto, rispettivamente, all'istotipo ed alla classe BI-RADS. Complessivamente, 65/84 (77,4\%) lesioni sono state correttamente classificate come benigne o maligne all'elastosonografia, mentre le rimanenti 19/84 (22,6\%) sono state erroneamente valutate. In particolare, $27 / 84(32,1 \%)$ lesioni - 10/56 (17,8\%) di quelle classificate all'ecografia di base come BI-RADS 3 e 17/28 (60,7\%) di quelle classificate come BI-RADS 4 - sono state considerate come maligne alla valutazione elastosonografica (Fig. 1). Tuttavia, tredici di queste lesioni (4 fibroadenomi, 3 lesioni fibrotiche, 2 aree di steatonecrosi, 2 adenosi, 2 cisti corpuscolate) sono risultate benigne al riscontro anatomo-patologico e dunque considerate dei falsi positivi (Fig. 2). Di contro, $57 / 84$ (67,9\%) lesioni hanno presentato un punteggio elastosonografico di benignità (Fig. 3). Sei di queste ultime, tuttavia, si sono dimostrate maligne alla valutazione istologica - 5 carcinomi e 1 sarcoma - e, di conseguenza, considerate falsi negativi (Fig. 4). Nella serie presa in esame ne derivano, per quanto attiene l'elastosonografia, tassi di sensibilità, specificità, VPP e VPN pari rispettivamente a 70\%, 79,6\%, 51,8\% e $89 \%$ (Tabella 4). Parallelamente, considerando come benigne le lesioni in classe ecografica BI-RADS 3 e maligne quelle in classe BI-RADS 4, la valutazione della sola ecografia convenzionale ha portato al riscontro di 14 falsi positivi e 6 falsi negativi, con valori di sensibilità, specificità, VPP $e$ VPN pari rispettivamente a $68,4 \%, 78,5 \%, 48,1 \%$ e $89,5 \%$. 
Table 2 Ultrasound (US) elastography scores for the 56 Breast Imaging Reporting and Data System 3 lesions

\begin{tabular}{|c|c|c|c|c|c|}
\hline & \multicolumn{5}{|l|}{ US elastography score } \\
\hline & 1 & 2 & 3 & 4 & 5 \\
\hline & Cold abscess (1) & Fibroadenoma (19) & Fibroadenoma (2) & IDC (3) & - \\
\hline & Corpuscular cysts (6) & Fat necrosis (1) & Tubular carcinoma (1) & Fat necrosis (2) & - \\
\hline & Granuloma (1) & Angioma (8) & Ductal carcinoma (1) & Fibroadenoma (4) & - \\
\hline & Mucinous carcinoma (1) & Fibrosis (1) & & Corpuscular cyst (1) & - \\
\hline & Fibroadenoma (2) & Corpuscular cysts (2) & & & - \\
\hline Total & 11 & 31 & 4 & 10 & - \\
\hline
\end{tabular}

The number of lesions is shown in brackets

$I D C$, invasive ductal carcinoma

Tabella 2 Punteggio elastosonografico delle 56 lesioni mammarie in classe BI-RADS 3

\begin{tabular}{|c|c|c|c|c|c|}
\hline & \multicolumn{5}{|l|}{ Punteggio elastosonografico } \\
\hline & 1 & 2 & 3 & 4 & 5 \\
\hline & Ascesso freddo (1) & Fibroadenoma (19) & Fibroadenoma (2) & $C D I(3)$ & - \\
\hline & Cisti corpuscolata (6) & Liponecrosi (1) & Carcinoma tubulare (1) & Liponecrosi (2) & - \\
\hline & Granuloma (1) & Angioma (8) & Carcinoma duttale (1) & Fibroadenoma (4) & - \\
\hline & Carcinoma mucinoso (1) & Fibrosi (1) & & Cisti corpuscolata (1) & - \\
\hline & Fibroadenoma (2) & Cisti corpuscolata (2) & & & - \\
\hline Totale & 11 & 31 & 4 & 10 & - \\
\hline
\end{tabular}

Tra parentesi è indicato il numero di lesioni

CDI, carcinoma duttale infiltrante

On B-mode US, 56/84 lesions (66.7\%) were classified as BI-RADS 3 and the remaining $28(33.3 \%)$ as BI-RADS 4. Tables 1-3 show the US elastography scores of the breast lesions in relation to histological type and BI-RADS category. Overall, 65/84 (77.4\%) lesions were correctly classified as benign or malignant at US elastography, whereas the remaining $19 / 84(22.6 \%)$ were incorrectly assessed. In particular, $27 / 84(32.1 \%)$ lesions - 10/56 (17.8\%) of those classified as BI-RADS 3 on B-mode US and 17/28 $(60.7 \%)$ of those classified as BI-RADS 4 - were classified as malignant at US elastography (Fig. 1). However, 13 of these lesions (four fibroadenomas, three fibrotic lesions, two areas of fat necrosis, two adenoses and two corpuscular cysts) proved to be benign on pathological examination and were therefore considered FP results (Fig. 2). In contrast, $57 / 84(67.9 \%)$ lesions had benign US elastography scores (Fig. 3). Six of them, however, proved to be malignant at histological assessment - five carcinomas and one sarcoma - and were therefore considered FN results (Fig. 4). Thus, in the series examined, US elastography had $70 \%$ sensitivity, 79.6\% specificity, 51.8\% PPV and $89 \%$ NPV (Table 4). In parallel, if we consider BI-RADS 3 lesions as benign and BI-RADS 4 lesions as malignant, assessment with conventional US alone yielded $14 \mathrm{FP}$ and six FN results, leading to sensitivity, specificity, PPV and NPV values of $68.4 \%$, $78.5 \%, 48.1 \%$ and $89.5 \%$, respectively.
L'indagine elastosonografica ha fornito un giudizio difforme rispetto all'ecografia di base in 19/84 (22,6\%) casi, modificando $i$ reperti ecografici B-mode iniziali dalla malignità verso la benignità in dieci casi e viceversa nei rimanenti nove. Inoltre, in dieci casi tale modificazione è risultata confermata dai reperti istologici, mentre in nove è risultata smentita dall'istologia. In particolare, sette lesioni benigne (2 granulomi su cicatrice, 2 fibroadenomi, 1 adenosi, 1 iperplasia pseudoangiomatosa, 1 cisti corpuscolata) classificate in classe BI-RADS 4 sono state correttamente valutate come benigne alla valutazione elastosonografica, mentre 3 lesioni maligne (tutti carcinomi duttali infiltranti), classificate in classe BI-RADS 3, sono state correttamente valutate come maligne all'indagine elastosonografica. Di contro, sei lesioni correttamente valutate in classe BI-RADS 3 (4 fibroadenomi e 2 aree di steatonecrosi) sono state erroneamente valutate come maligne alla valutazione elastosonografica. Infine, tre lesioni maligne (1 carcinoma tubulare, 1 carcinoma duttale ascessualizzato e 1 sarcoma) classificate come BI-RADS 4 sono state invece erroneamente considerate benigne alla valutazione elastosonografica.

\section{Classe ecografica BI-RADS 3}

Nella Tabella 2 sono riportati i punteggi relativi alla valutazione elastosonografica delle 56 lesioni mammarie in classe 
Table 3 Ultrasound (US) elastography scores for the 28 Breast Imaging Reporting and Data System category 4 breast lesions

\begin{tabular}{|c|c|c|c|c|c|}
\hline & \multicolumn{5}{|l|}{ US elastography score } \\
\hline & 1 & 2 & 3 & 4 & 5 \\
\hline & \multirow[t]{6}{*}{ Ductal carcinoma (1) } & Granuloma (2) & Adenosis (1) & $\operatorname{IDC}(5)$ & $\operatorname{IDC}(3)$ \\
\hline & & Sarcoma (1) & Tubular carcinoma (1) & Ductal carcinomain situ (1) & - \\
\hline & & Fibroadenoma (2) & $\begin{array}{l}\text { Pseudoangiomatous } \\
\text { hyperplasia (1) }\end{array}$ & Lobular carcinoma (2) & - \\
\hline & & Adenosis (1) & Corpuscular cyst (1) & Adenosis (2) & - \\
\hline & & & & Fibrosis (3) & - \\
\hline & & & & Corpuscular cyst (1) & \\
\hline Total & 1 & 6 & 4 & 14 & 3 \\
\hline
\end{tabular}

The number of lesions is shown in brackets $I D C$, invasive ductal carcinoma

Tabella 3 Punteggio elastosonografico delle 28 lesioni mammarie in classe BI-RADS 4

\begin{tabular}{|c|c|c|c|c|c|}
\hline & \multicolumn{5}{|c|}{ Punteggio elastosonografico } \\
\hline & 1 & 2 & 3 & 4 & 5 \\
\hline & \multirow[t]{6}{*}{ Carcinoma duttale (1) } & Granuloma (2) & Adenosi (1) & $C D I(5)$ & $C D I(3)$ \\
\hline & & Sarcoma (1) & Carcinoma tubulare (1) & Carcinoma duttale in situ (1) & - \\
\hline & & Fibroadenoma (2) & $\begin{array}{l}\text { Iperplasia } \\
\text { pseudoangiomatosa (1) }\end{array}$ & Carcinoma lobulare (2) & - \\
\hline & & Adenosi (1) & Cisti corpuscolata (1) & Adenosi (2) & - \\
\hline & & & & Fibrosi (3) & - \\
\hline & & & & Cisti corpuscolata (1) & \\
\hline Totale & 1 & 6 & 4 & 14 & 3 \\
\hline
\end{tabular}

Tra parentesi è indicato il numero di lesioni

CDI, carcinoma duttale infiltrante

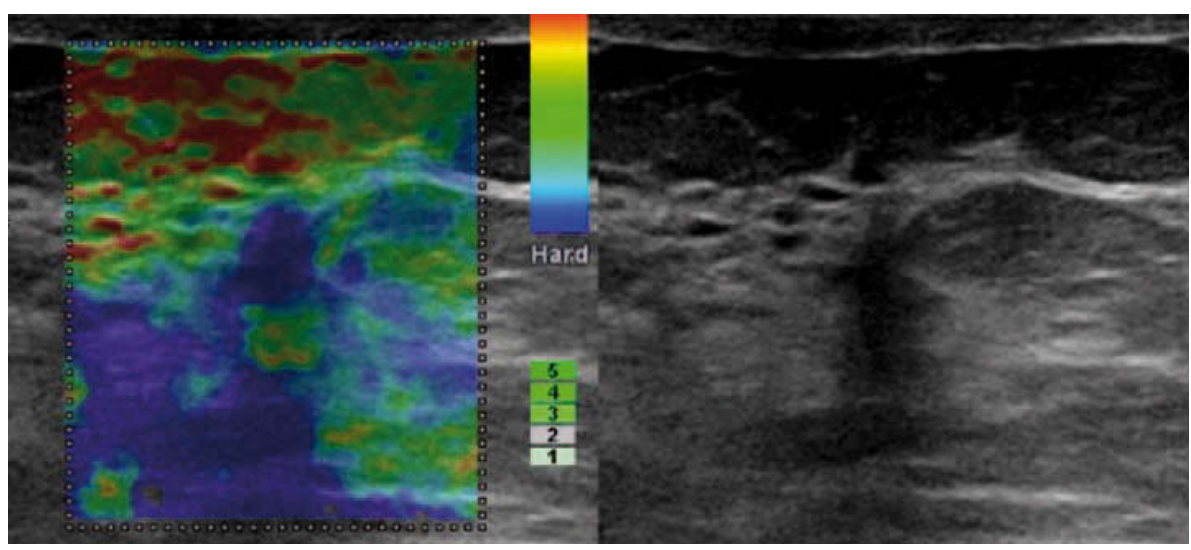

Fig. 1 Invasive ductal carcinoma in a 65-yearold woman. B-mode ultrasound (US) (right) depicts a heterogeneous hypoechoic lesion with irregular margins and a diameter of 6 $\mathrm{mm}$ (Breast Imaging Reporting and Data System US category 4). On the left, US elastogram shows marked stiffness of the entire lesion (score 5).

Fig. 1 Carcinoma duttale infiltrante in donna di 65 anni. All'ecografia di base (destra) si apprezza una lesione ipoecogena a margini irregolari del diametro di $6 \mathrm{~mm}$ (classe BI-RADS ecografica 4). A sinistra, la valutazione elastosonografi a dimostra una marcata rigidità tissutale di tutta la lesione (punteggio 5).

US elastography led to a different judgement from that obtained using B-mode US in 19/84 (22.6\%) cases, changing the initial B-mode US findings from benign to malignant in ten cases and from malignant to benign in the remaining nine. The change was confirmed by the histological assessment in 10 cases and refuted in nine. In particular, seven benign lesions (two granulomas on scars, two fibroadenomas, one adenosis, one pseudoan- ecografica BI-RADS 3. Nessuna di queste ha presentato un punteggio elastosonografico pari a 5, tuttavia 10/56 (17,9\%) di queste ultime hanno presentato un punteggio elastosonografico pari a 4, deponente quindi per malignità. Tale giudizio è stato confermato dall'esame istologico in tre casi di carcinoma duttale infiltrante, ma sconfessato nei rimanenti sette casi (4 fibroadenomi, 2 aree di steatonecrosi, 1 cisti corpuscolata). Per quanto attiene le 46 lesioni classificate in 

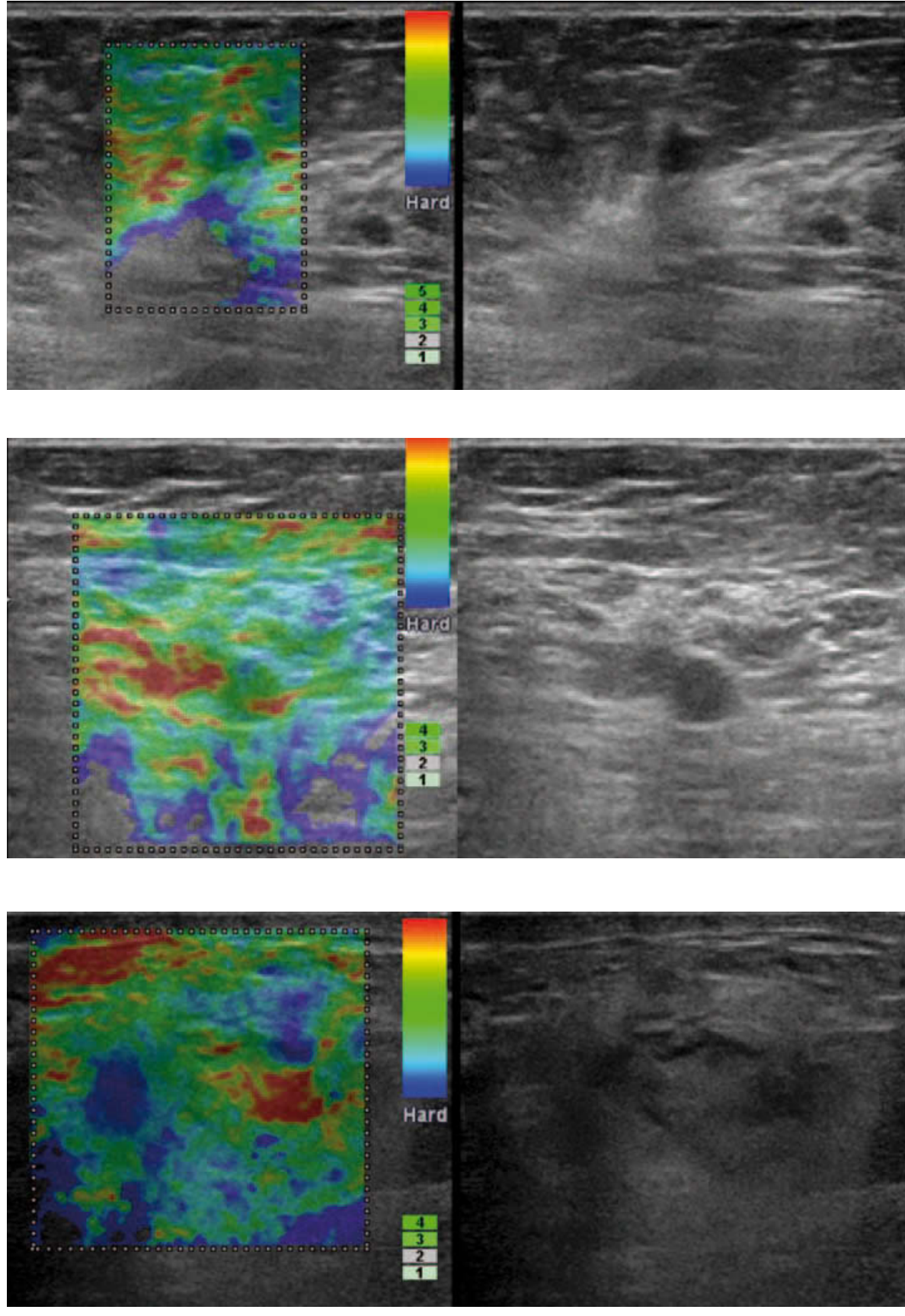

Fig. 2 Fibrosis in a 40-year-old woman B-mode ultrasound (US) (right) shows a heterogeneous hypoechoic lesion with a diameter of $6 \mathrm{~mm}$ (Breast Imaging Reporting and Data System US category 4). On the left, US elastogram shows marked stiffness of the entire lesion (score 4).

Fig. 2 Area di fibrosi in donna di 40 anni. All'ecografia di base (destra) si apprezza una lesione ipoecogena a margini irregolari del diametro di $6 \mathrm{~mm}$ (classe BI-RADS ecografica 4). A sinistra, la valutazione elastosonografica dimostra una marcata rigidità tissutale di tutta la lesione (punteggio 4).

Fig. 3 Cystic lesion in a 51-year-old woman. B-mode ultrasound (US) (right) depicts a homogeneously hypoechoic lesion without posterior acoustic enhancement showing regular margins, $5 \mathrm{~mm}$ in diameter (Breast Imaging Reporting and Data System US category 3 ). On the left, US elastogram shows the diffuse elasticity of the lesion with a partially trilaminar appearance (score 1).

Fig. 3 Cisti corpuscolata in donna di 51 anni. All'ecografia di base (destra) si apprezza una lesione ipoecogena a margini regolari del diametro di $5 \mathrm{~mm}$ (classe BI-RADS ecografica 3). A sinistra, la valutazione elastosonografica dimostra la diffusa elasticità della lesione con parziale aspetto trilaminare (punteggio 1).

Fig. 4 Abscessed invasive ductal carcinoma in a 55-year-old woman. B-mode ultrasound (US) (right) depicts an isohypoechoic lesion with ill-defined margins and a diameter of $15 \mathrm{~mm}$ (Breast Imaging Reporting and Data System US category 4). On the left, US elastogram shows a predominantly elastic lesion with a partially trilaminar appearance (score 1).

Fig. 4 Carcinoma duttale infiltrante ascessualizzato in donna di 55 anni. All'ecografia di base (destra) si apprezza una lesione iso-ipoecogena a margini mal definiti del diametro di $15 \mathrm{~mm}$ (classe BI-RADS ecografica 4). A sinistra, la valutazione elastosonografica dimostra la diffusa elasticità della lesione con parziale aspetto trilaminare (punteggio 1). giomatous hyperplasia and one corpuscular cyst) classified as BI-RADS 4 were correctly assessed as benign at US elastography, whereas three malignancies (all ductal cancers) classified as BI-RADS 3 were correctly assessed as malignant at US elastographic. In contrast, six lesions correctly assessed as BI-RADS 3 (four fibroadenomas and two areas of fat necrosis) were incorrectly assessed as malignant at US elastography. Finally, three malignancies (one tubular carcinoma, one abscessed ductal carcinoma and one sarcoma) classified as BI-RADS 4 were instead erroneously considered benign at US elastography. classe BI-RADS 3 e con punteggio elastosonografico di benignità (da 1 a 3), tre carcinomi (1 mucinoso, 1 tubulare e 1 duttale infiltrante) hanno presentato un punteggio elastosonografico di benignità, pari rispettivamente a 1 per il primo e 3 per i rimanenti due. Applicando, dunque, il relativo sistema di punteggio alla classe BI-RADS 3 l'elastosonografia ha presentato valori di sensibilità, specificità, VPP e VPN pari rispettivamente a 50\%, 86\%, 30\% e 93,5\% (Tabella 4).

\section{Classe ecografica BI-RADS 4}

Nella Tabella 3 sono riportati i punteggi relativi alla valu- 
Table 4 Sensitivity, specificity and positive and negative predictive values of ultrasound (US) elastography, overall and relative to the Breast Imaging Reporting and Data System (BI-RADS) US classification. Values are expressed as percentages (95\% CI)

US elastography

\begin{tabular}{lllll}
\hline & All lesions & BI-RADS 3 & BI-RADS 4 & BI-RADS3 vs. BI-RADS4* \\
\hline Sensitivity & $70.0(49.9-90.1)$ & $50.0(28.1-71.9)$ & $78.6(60.6-96.6)$ & 0.058 \\
Specificity & $79.6(69.7-89.57)$ & $86.0(77.5-94.5)$ & $57.1(45.0-69.2)$ & 0.000 \\
PPV & $51.8(33.0-70.7)$ & $30.0(12.7-47.3)$ & $64.7(46.7-82.7)$ & 0.011 \\
NPV & $89(80.9-97.1)$ & $93.5(87.1-99.9)$ & $72.7(61.1-84.3)$ & 0.003 \\
\hline
\end{tabular}

$P P V$, positive predictive value; $N P V$, negative predictive value $* p$ value

Tabella 4 Sensibilità, specificità, valori predittivi positivo e negativo dell'elastosonografia, complessivamente e in rapporto alla classe breast imaging reporting and data system (BI-RADS) ecografica. I valori sono espressi in percentuale (95\% CI)

\begin{tabular}{lllll}
\hline \multicolumn{7}{c}{ Elastosonografia } & & & \\
\hline & Tutte le lesioni & BI-RADS 3 & BI-RADS 4 & BI-RADS3 vs. BI-RADS4* \\
\hline Sensibilità & $70,0(49,9-90,1)$ & $50,0(28,1-71,9)$ & $78,6(60,6-96,6)$ & 0,058 \\
Specificità & $79,6(69,7-89,57)$ & $86,0(77,5-94,5)$ & $57,1(45,0-69,2)$ & 0,000 \\
$V P P$ & $51,8(33,0-70,7)$ & $30,0(12,7-47,3)$ & $64,7(46,7-82,7)$ & 0,011 \\
$V P N$ & $89(80,9-97,1)$ & $93,5(87,1-99,9)$ & $72,7(61,1-84,3)$ & 0,003 \\
\hline
\end{tabular}

$V P P$, valore predittivo positivo; VPN, valore predittivo negativo

$* p$

\section{BI-RADS US category 3}

Table 2 shows the US elastography scores of 56 BI-RADS US category 3 lesions. Although none of them had a US elastography score of $5 ; 10 / 56(17.9 \%)$ had a score of 4 , suggesting malignancy. This assessment was confirmed by histology in three cases of invasive ductal carcinoma but refuted in the remaining seven cases (four fibroadenomas, two areas of fat necrosis and one case of corpuscular cyst). Among the remaining 46 BI-RADS 3 lesions with benign US elastography scores (1-3), there were three carcinomas: one mucinous (score 1), one tubular (score 3 ) and one invasive ductal carcinoma (score 3). Therefore, applying the relevant scoring system to BI-RADS 3 lesions, US elastography had sensitivity, specificity, PPV and NPV of 50\%, $86 \%, 30 \%$ and $93.5 \%$, respectively (Table 4 ).

\section{BI-RADS US category 4}

Table 3 shows the US elastography scores of the 28 BI-RADS US category 4 lesions. In particular, 17/28 $(60.7 \%)$ had US elastography scores suggestive of malignancy (4 or 5). This was confirmed by histology in 11 cases but refuted in the remaining six (three cases of fibrosis, one adenosis, one intracystic mass and one simple ductal hyperplasia). In contrast, among the 11 BI-RADS 4 lesions with benign US elastography scores (1-3), histology revealed tazione elastosonografica delle 28 lesioni mammarie in classe ecografica BI-RADS 4. In particolare, 17/28 (60,7\%) di queste hanno presentato un punteggio elastosonografico di malignità (4 o 5), confermata in 11 casi all'istologia, ma smentita nei rimanenti sei casi (3 fibrosi, 1 adenosi, 1 neoformazione intracistica e 1 iperplasia duttale semplice). Di contro, tra le 11 lesioni in classe BI-RADS 4 con punteggio elastosonografico di benignità (1-3) l'esame istologico ha confermato la presenza di 1 carcinoma ascessualizzato, 1 sarcoma e 1 carcinoma tubulare, rispettivamente presentanti all'indagine elastosonografica un punteggio di 1, 2 e 3. Di conseguenza, applicando il relativo sistema di punteggio alla classe BI-RADS 4, l'elastosonografia ha presentato valori di sensibilità, specificità, VPP e VPN pari rispettivamente a 78,6\%, 57,1\%, 64,7\% e 72,7\% (Tabella 4).

\section{Discussione}

In accordo con quanto riportato da alcuni autori, nel nostro studio, mirato alla valutazione del ruolo dell'elastosonografia nelle lesione focali mammarie indeterminate all'ecografia di base, non abbiamo riscontrato differenze statisticamente significative tra le due metodiche in termini di sensibilità, specificità e valori predittivi, positivo e negativo [12, 13]. Complessivamente, nella nostra esperienza, l'elastosonografia ha dimostrato una sensibilità del $70 \%$, infe- 
the presence of one abscessed carcinoma, one sarcoma and one tubular carcinoma, with US elastography scores of 1,2 and 3, respectively. Therefore, applying the relevant scoring system to BI-RADS category 4 lesions, US elastography had sensitivity, specificity, PPV and NPV of 78.6\%, 57.1\%, $64.7 \%$ and $72.7 \%$, respectively (Table 4 ).

\section{Discussion}

Consistent with previous reports, our study, which aimed at evaluating the role of US elastography in characterising focal breast lesions classified as indeterminate on B-mode US, found no statistically significant differences between the two techniques in terms of sensitivity, specificity, PPV or NPV [12, 13]. Overall, in our experience, US elastography had a sensitivity of $70 \%$, lower than reported by other authors (79-96.9\%) and a specificity of $79.6 \%$, within the range reported in the literature $(76-89 \%)[8,12$, $14,15]$. These results can be, at least in part, explained by the different composition of the patient series examined. In fact, after excluding BI-RADS US category 5 lesions from our analysis (for which biopsy is recommended), the prevalence of malignant lesions amounted to $33.8 \%$, significantly lower than reported in other studies, which had peaks of $76 \%[6,8,12]$. One study only, with a $33.2 \%$ prevalence of malignant lesions, reported a sensitivity of $96.9 \%$, but this was obtained using a different US elastography scoring system that is not directly comparable with the one adopted by us [15]. However, if we analyse data for the two BI-RADS categories separately, the specificity of US elastography appears appreciably greater in BI-RADS 3 lesions when compared with BI-RADS 4 (86\% vs. $57.1 \%$ ), in agreement with previous findings with respect to BI-RADS mammography categories [8]. Rather than being due to any real difference in the diagnostic power of the technique with regard to benign or malignant lesions, these results can be primarily ascribed to the greater prevalence of malignancies among BI-RADS 4 lesions compared with BI-RADS 3 lesions, with a consequent direct statistical effect on the significance of the relevant rates, as demonstrated by the consensual differences in sensitivity $(50 \%$ vs. $78.6 \%)$ and PPV (30\% vs $64.7 \%)$ and NPV (93.5\% vs. $72.7 \%$ ) for BI-RADS 3 and 4 lesions, respectively. In the case of indeterminate BI-RADS 3 breast lesions, our findings, in agreement with the literature, support the use of US elastography for its high NPV and therefore its ability to confirm the benign nature of the lesion and thus avoid the need for biopsy $[8,16,17]$. In particular, in our experience, US elastography proved particularly useful in corpuscular cysts, which passed from a score of BI-RADS 3 (probably benign) to a US elastography score of 1 (highly suggestive of benignity). In this regard, it should also be noted that riore ai valori riportati da altri autori - variabili tra il $79 \%$ ed il 96,9\% - e una specificità del 79,6\%, compresa nell'ambito dei valori riportati in letteratura $(76 \%-89 \%)[8,12$, 14, 15]. Tali risultati risultano, almeno in parte, spiegabili con la diversa composizione del campione preso in esame. Infatti, avendo escluso dalla nostra analisi la classe ecografica BI-RADS 5, per la quale il campionamento bioptico è già indicato, la prevalenza di lesioni maligne è risultata, nella nostra casistica, pari al 33,8\%, dunque nettamente inferiore a quella di altri studi, che presentavano punte anche del $76 \%[6,8,12]$. Solo in uno studio, con prevalenza di lesioni maligne pari al 33,2\%, si è riscontrata una sensibilità pari al 96,9\%, ma impiegando un punteggio elastosonografico diverso e quindi non direttamente comparabile a quello usato nel presente lavoro [15]. Tuttavia, analizzando separatamente $i$ dati attinenti alle due classi ecografiche BI-RADS da noi prese in esame, risulta apprezzabile la maggiore specificità dell'elastosonografia nella valutazione delle lesioni in classe BI-RADS 3 rispetto a quelle BI-RADS $4(86 \%$ vs. 57,1\%), in accordo a quanto già riportato in letteratura rispetto alle classi BI-RADS mammografiche [8]. Tali risultati sono primariamente correlabili, più che a un reale differente potere diagnostico della metodica in relazione alla natura benigna o maligna della lesione, all'incremento della prevalenza delle lesioni maligne nella classe BI-RADS 4 rispetto alla BI-RADS 3, con conseguente effetto statistico diretto sulla significatività dei tassi correlati, come dimostrato dalle consensuali variazioni della sensibilità $(50 \%$ vs. $78,6 \%)$ e dei valori predittivi positivo $(30 \%$ vs. $64,7 \%)$ e negativo $(93,5 \%$ vs. $72,7 \%)$ rispettivamente per la classe BI-RADS 3 e 4. Nel caso, dunque, di lesione mammaria indeterminata in classe ecografica BI-RADS 3 , la valutazione statistica dei nostri dati, in accordo a quanto riportato in letteratura, supporta l'impiego dell'elastosonografia per il suo elevato VPN e, quindi, per confermare la natura benigna di una lesione, evitando il ricorso alla biopsia [8, 16, 17]. Nella nostra esperienza, in particolare, il contributo della valutazione elastosonografica è risultato particolarmente utile nel caso delle cisti corpuscolate, passate nella quasi totalità dei casi da un punteggio BI-RADS 3, pur indicativo di probabile benignità, ad uno elastosonografico 1 , altamente suggestivo di benignità. A tale proposito, appare opportuno ricordare come anche l'analisi color Doppler possa fornire utili indicazione al fine di distinguere le cisti non tumorali dai tumori mammari intracistici [18]. Considerazioni analoghe possono essere fatte riguardo le lesioni angiomatose, costantemente classificate come benigne all'analisi elastosonografica, con punteggio pari a 2. Tuttavia, tre carcinomi classificati come BI-RADS 3 (1 mucinoso, 1 tubulare e 1 duttale infiltrante) hanno presentato, nella nostra serie, un punteggio elastosonografico di benignità, almeno in parte da mettere in relazione alle caratteristiche istologiche, quali l'elevato contenuto di mucina, o alle dimensioni superiori a 2 centimetri, situazione quest'ultima che può condizionare la diso- 
colour Doppler can help to distinguish nontumoural cysts from intracystic breast carcinomas [18]. Similar considerations can be made regarding angiomatous lesions, which were constantly classified as benign, with a US elastography score of 2. However, three carcinomas classified as BI-RADS 3 (one mucinous, one tubular and one invasive ductal) had a benign US elastography score, at least in part as a result of histological features such as high mucin content, or a size $>2 \mathrm{~cm}$ that can lead to lesion heterogeneity due the presence of necrotic-colliquative areas, as demonstrated by Giuseppetti et al. [6]. In this regard, it would seem appropriate to periodically follow-up BI-RADS 3 lesions not subjected to biopsy in order to assess their stability over time and thus lower the risk of missing the opportunity of early diagnosis of a possible malignancy [19]. In contrast, the PPV of US elastography in BI-RADS 4 focal breast lesions $-64.7 \%$ in our experience - is not sufficiently high to the need for biopsy on the basis of the US elastography score alone, as suggested by a previous report on nonpalpable breast lesions [12]. In our series, two carcinomas and one sarcoma classified as BI-RADS 4 had a benign US elastography score. In particular, one of the two carcinomas showed abundant inflammatory infiltrate, a histological feature that can significantly increase elasticity. In addition, although all BI-RADS 4 lesions with a US elastography score of 5 proved to be ductal carcinomas at histological examination, six of the 14 BI-RADS 4 lesions with a US elastography score of 4 turned out to be benign. In particular, we found areas of fibrosis to be especially difficult to characterise correctly with US elastography. This can be explained by the lack of elasticity of scar tissue, with the resulting difficulty in differentiating fibrosis from carcinoma. This is confirmed by the fact that an abundant fibrotic component accounted for a malignant US elastography score in four fibroadenomas in our series, whereas the majority of fibroadenomas $(25 / 29,86 \%)$ had a high level of elasticity and consequently a benign US elastography score.

There are several limitations to our study. First, the reference standard was not histological examination of the surgical specimen in all cases, but the semiological criteria used in cyto-microhistological evaluation, known in the literature and commonly used in clinical practice. Second, the readers evaluated the images in consensus and it was therefore not possible to assess the degree of agreement between independent readers. In addition, as all US elastography examinations were performed by a single operator, it was not possible to assess the interoperator variability of this relatively new US technique. Further research is needed to evaluate these aspects.

In conclusion, by providing additional diagnostic information regarding the elasticity of breast lesions, US elastography completes but does not in any way replace the mogeneità della lesione per la presenza di aree necroticocolliquative, come già dimostrato da Giuseppetti et al. [6]. A tale proposito, appare opportuno controllare periodicamente le lesioni classificate come BI-RADS 3, ancorché non sottoposte a biopsia, al fine valutarne la stabilità nel tempo $e$, quindi, di ridurre la possibilità di non diagnosticare tempestivamente una eventuale lesione maligna [19]. Di contro, il VPP riportato dall'elastosonografia nell'analisi delle lesioni focali mammarie BI-RADS 4, pari nella nostra esperienza al $64,7 \%$, non risulta abbastanza soddisfacente da consentire di escludere il ricorso alla biopsia sulla scorta del solo punteggio elastosonografico, in accordo con quanto suggerito da un precedente studio condotto su lesioni mammarie non palpabili [12]. Nella nostra serie, due carcinomi e un sarcoma in classe BI-RADS 4 hanno presentato un punteggio elastosonografico di benignità. In particolare, uno dei due carcinomi presentava un considerevole infiltrato flogistico, caratteristica istologica in grado di aumentarne considerevolmente l'elasticità. Inoltre, sebbene tutte le lesioni in classe BI-RADS 4 con punteggio elastosonografico pari a 5 si siano rivelate dei carcinomi duttali all'indagine istologica, sei delle 14 lesioni focali in classe BI-RADS 4 con punteggio elastosonografico pari a 4 si sono rivelate in realtà benigne. In particolare, nella nostra esperienza, le aree di fibrosi si sono rilevate particolarmente difficili da caratterizzare come tali all'indagine elastosonografica. Tale risultato è spiegabile con la poca elasticità del tessuto cicatriziale, con la conseguente difficoltà di operare la diagnosi differenziale tra fibrosi e carcinoma. A conferma di quanto appena esposto, proprio la presenza di abbondante componente fibrotica è stata alla base del riscontro di un punteggio elastosonografico di malignità in quattro fibroadenomi della nostra serie. Questi ultimi, di contro, hanno presentato nella maggior parte dei casi $(25 / 29,86 \%)$ un'elavata elasticità $e$, di conseguenza, un punteggio elastosonografico di benignità.

Il nostro studio presenta delle limitazioni. In primo luogo lo standard di riferimento non è stato, in tutti i casi, la valutazione istologica del resecato operatorio, bensì $i$ criteri semeiologici alla valutazione cito-microistologia, ancorché noti in letteratura e comunemente impiegati nella pratica clinica. Inoltre, da parte dei lettori è stata effettuata una valutazione in consenso e, pertanto, non è stato possibile valutare il grado di correlazione tra lettori indipendenti. Inoltre, un solo operatore ha effettuato tutti gli esami elastosonografici e, pertanto, non è stato possibile valutare la variabilità interoperatore di una metodica di impiego relativamente recente come l'elastosonografia. Ulteriori studi sono auspicabili per valutare tali aspetti.

In conclusione, nella nostra esperienza, l'elastosonografia, fornendo ulteriori informazioni diagnostiche circa l'elasticità delle lesioni mammarie, completa ma non sostituisce in alcun modo la valutazione morfologica dell'esame ecografico in B-mode. In particolare, l'elevato valore predittivo negativo dell'elastosonografia può essere utile 
morphological evaluation of B-mode US. In particular, the high NPV of US elastography may help to avoid the extensive use of biopsy for BI-RADS 3 lesions, but its relatively low PPV in BI-RADS 4 lesions does not allow biopsy to be omitted on the basis of US elastography scores alone in this category of lesions. per evitare l'estensivo ricorso alla biopsia nelle lesioni mammarie in classe BI-RADS 3, ma il suo relativamente basso valore predittivo positivo nella valutazione delle lesioni in classe BI-RADS 4 non consente, in quest'ultimo ambito, di escludere il ricorso alla biopsia semplicemente sulla scorta del solo punteggio elastosonografico.

\section{Conflict of interest None}

\section{References/Bibliografia}

1. Del Frate C, Bestagno A, Cerniato R et al (2006) Sonographic criteria for differentiation of benign and malignant solid breast lesions: size is of value. Radiol Med 111:783-796

2. Costantini M, Belli P, Ierardi C et al (2007) Solid breast mass characterisation: use of the sonographic BI-RADS classification. Radiol Med 112:877-894

3. Abdullah N, Mesurolle B, El-Khoury M, Kao E (2009) Breast imaging reporting and data system lexicon for US: interobserver agreement for assessment of breast masses. Radiology 252:665-672

4. Garra BS, Cespedes EI, Ophir J et al (1997) Elastography of breast lesions: initial clinical results. Radiology 202:79-86

5. Konofagou E, Ophir J (1998) A new elastographic method for estimation and imaging of lateral displacements, lateral strains, corrected axial strains and Poisson's ratios in tissues. Ultrasound Med Biol 24:1183-1199

6. Giuseppetti GM, Martegani A, Di Cioccio B, Baldassarre S (2005) Elastosonography in the diagnosis of the nodular breast lesions: preliminary report. Radiol Med 110:69-76
7. Itoh A, Ueno E, Tohno E et al (2006) Breast disease: clinical application of US elastography for diagnosis. Radiology 239:341-350

8. Thomas A, Kümmel S, Fritzsche F et al (2006) Real-time sonoelastography performed in addition to B-mode ultrasound and mammography: improved differentiation of breast lesions? Acad Radiol 13:1496-1504

9. Cho N, Moon WK, Park JS (2009) Real-time US elastography in the differentiation of suspicious microcalcifications on mammography. Eur Radiol 19:1621-1628

10. Zhu QL, Jiang YX, Liu JB et al (2008) Real-time ultrasound elastography: its potential role in assessment of breast lesions. Ultrasound Med Biol 34:12321238

11. D’Orsi CJ, Bassett LW, Berg WA et al (2003) Breast Imaging Reporting and Data System: ACR BI-RADSMammography (4th edn). American College of Radiology, Reston

12. Scaperrotta G, Ferranti C, Costa C et al (2008) Role of sonoelastography in non-palpable breast lesions. Eur Radiol 18:2381-2389

13. Sohn YM, Kim MJ, Kim EK et al (2009) Sonographic elastography combined with conventional sonography: how much is it helpful for diagnostic performance? J Ultrasound Med 28:413-420
14. Raza S, Odulate A, Ong EM et al (2010) Using real-time tissue elastography for breast lesion evaluation: our initial experience. J Ultrasound Med 29:551-563

15. Schaefer FK, Heer I, Schaefer PJ et al (2009) Breast ultrasound elastography - Results of 193 breast lesions in a prospective study with histopathologic correlation. Eur J Radiol, in press

16. Regini E, Bagnera S, Tota D et al (2010) Role of sonoelastography in characterising breast nodules. Preliminary experience with 120 lesions. Radiol Med 115:551-562

17. Wojcinski S, Farrokh A, Weber S et al (2010) Multicenter study of ultrasound real-time tissue elastography in 779 cases for the assessment of breast lesions: improved diagnostic performance by combining the BIRADS(R)-US classification system with sonoelastography. Ultraschall Med 31:484-491

18. Catalano O, Raso MM, D'Aiuto M et al (2009) Additional role of colour Doppler ultrasound imaging in intracystic breast tumours. Radiol Med 114:253-266

19. Jellici E, Minniti S, Malagò R et al (2006) Focal breast lesions with benign appearances. Review of eight breast cancers with initial features of intramammary lymph node. Radiol Med 111:1078-1086 